\title{
Tendinopathy: no longer a 'one size fits all' diagnosis
}

\section{Jill Cook}

Tendon research has come a long way. So far, in fact, that 120 tendon-interested clinicians and scientists met in Umeå, north Sweden, in October 2010 at a conference called 'Neuronal and non-neuronal pathways in the tendon pathology continuum'. The novel data, vigorous exchanges and the generation of new ideas for study highlighted the buzz in this field. The stories of challenging clinical cases underscored the urgency for improvements in diagnosis and in treatment. In this issue, you will find papers on new surgical techniques (see pages 407 and 411), advances in understanding tendon pain (see pages 393 and 429) and updates on injection therapies (see pages 411 and 424). All these allow you to manage patients and athletes who present with problematical tendon symptoms more effectively.

\section{SUBGROUPS, STAGES AND ALGORITHMS}

One of the issues arising from all this research is that the days are well gone when tendinopathy was an overarching diagnosis. Subgroups of tendinopathies, different stages of presentation, and risk factors that vary between sexes, ages and activities mean that the diagnosis must be more specific than just 'tendinopathy'.

This is most clearly described by Professor Alfredson (see pages 386 and 407), who raises the concept of plantaris being the root of some failed Achilles tendinopathy treatments. Although he suggests a surgical solution (he is a surgeon!) there are conservative approaches that may work if applied in the early stages. As he suggests that compression of the plantaris against the Achilles may be a key aetiology, then reducing compression similar to the approach taken with insertional Achilles tendon problems can help. ${ }^{1}$ It may also explain why heel raises help some people with Achilles tendinopathy and yet do not seem to help others.

On top of building this into your diagnostic algorithm, you can add another

Correspondence to Dr Jill Cook, Monash University, McMahons Road, Frankston, VIC 3199, Australia; jill.cook@monash.edu finding from the same group; the possibility that the Achilles tendon can have superficial tears. Certainly it puts the onus of accurate diagnosis as a key part of your clinical pathway, as treatments can vary greatly. Achilles tendinopathy is no longer an uncomplicated diagnosis with eccentric exercises as the universal answer.

\section{ADVANCES IN TREATMENT}

Although diagnosis is a cornerstone of clinical practice, advances in treatment are what most clinicians want to hear about. Simplistic and unimodal interventions have produced varied outcomes and this is also evident in this issue. Platelet-rich plasma, ${ }^{2}$ one could be tempted to call a fashionable or funky treatment for tendons, ${ }^{3}$ promised to deliver a cocktail of appropriate growth factors to the tendinopathy region and improve both pain and tendon healing. A randomised trial has shown that it was no better than saline for pain, ${ }^{4}$ and did not improve ultrasound appearance, a surrogate measure for pathology (see page 387 ).

Prolotherapy, on the other hand, added a small advantage over eccentric exercise alone in the short term as shown in another randomised trial (see page 421). Similarly in the elbow, although shortterm effects of glyceryl trinitrate patches have been described, ${ }^{5}$ the longer-term outcome is no better than standard treatment (see page 416). These treatments may be especially beneficial in athletes when quick recovery is an essential part of your decision making.

The complexities of making treatment decisions in tendinopathy is enhanced by these well-designed and well run trials. What we now need to know is the effect of the diagnostic subgroups mentioned above; would someone with a plantaris tendon or a superficial tear be less likely to be responsive to injection therapies? Every time you think you are getting close to understanding a simple presentation such as Achilles tendinopathy, more information to complicate your clinical processes is published.
As a clinically focused journal, BJSM only publishes animal studies that are of particular relevance to patients in the short term and the rabbit research in this issue does shed light on human tendinopathy. Commonly, individuals have unilateral symptoms, although often bilateral pathology is evident on imaging. This seems logical in the lower limb, but it also holds true in the upper $\operatorname{limb}^{6}$ where it makes less sense. Anderson et al (see page 399) show that loading one Achilles tendon produced pathological changes in the other tendon that was not loaded. Logically, the authors propose that there may be some central mechanisms involved, and this may clearly play a role in the bilateral pathology and/ or symptoms that present clinically. The 'global response' of tendons to stimuli is also reported when pain is induced experimentally (see page 393). Here the authors describe a decrease in muscle response in not only the calf muscle, but the agonists as well, when the tendon was painful.

So, another raft of new concepts to add to your clinical armour. There is no hint that Moses provided tendon solutions on a tablet, but tendon injury has been recognised as a major clinical problem and many excellent research groups throughout the world are sharing their best research via BJSM. Please do make suggestions as to what you would like us to address on $B J S M$ podcasts and tell us who you would like to hear from (jill.cook@monash.edu).

Provenance and peer review Not commissioned; not externally peer reviewed.

Accepted 18 January 2011

Br J Sports Med 2011;45:385. doi:10.1136/bjsm.2011.084111

\section{REFERENCES}

1. Jonsson P, Alfredson H, Sunding K, et al. New regimen for eccentric calf-muscle training in patients with chronic insertional Achilles tendinopathy: results of a pilot study. Br J Sports Med 2008;42:746-9.

2. Engebretsen L, Steffen K, Alsousou J, et al. IOC Consensus paper on the use of platelet-rich plasma in sports medicine. Br J Sports Med 2010;44:1072-81.

3. Cook J. Funky treatments in elite sports people: do they just buy rehabilitation time? Br J Sports Med 2010;44:221.

4. de Vos RJ, Weir A, van Schie HT, et al. Platelet-rich plasma injection for chronic Achilles tendinopathy: a randomized controlled trial. JAMA 2010;303:144-9.

5. Paoloni JA, Appleyard RC, Nelson J, et al. Topical nitric oxide application in the treatment of chronic extensor tendinosis at the elbow: a randomized, double-blinded, placebo-controlled clinical trial. Am J Sports Med 2003;31:915-20.

6. Miniaci A, Dowdy PA, Willits KR, et al. Magnetic resonance imaging evaluation of the rotator cuff tendons in the asymptomatic shoulder. Am J Sports Med 1995;23:142-5. 